OPEN ACCESS

Edited by:

Shiv K. Gupta,

Mayo Clinic, United States

Reviewed by:

Subhas Mukheriee,

Northwestern University, United States

Sonja Dragojevic

Mayo Clinic, United States

*Correspondence:

Hui Zhu

drzhuh@126.com

Jinming Yu

sdyujinming@163.com

†These authors have contributed equally to this work

Specialty section

This article was submitted to

Cancer Molecular Targets and

Therapeutics,

a section of the journal

Frontiers in Oncology

Received: 06 September 2020

Accepted: 15 February 2021

Published: 17 May 2021

Citation:

Li J, Jing W, Jia W, Zhai X, Zhu H and Yu J (2021) Downregulation of IncRNA XR_429159.1 Linked to Brain

Metastasis in Patients With

Limited-Stage Small-Cell Lung Cancer. Front. Oncol. 11:603271. doi: 10.3389/fonc.2021.603271

\section{Downregulation of IncRNA} XR_429159.1 Linked to Brain Metastasis in Patients With Limited-Stage Small-Cell Lung Cancer

\author{
Ji Li ${ }^{1 \dagger}$, Wang Jing ${ }^{2 \dagger}$, Wenxiao Jia ${ }^{2}$, Xiaoyang Zhai ${ }^{2}$, Hui Zhu ${ }^{2 *}$ and Jinming $\mathrm{Yu}^{1 *}$ \\ ${ }^{1}$ Department of Oncology, Renmin Hospital of Wuhan University, Wuhan, China, ${ }^{2}$ Department of Radiation Oncology, \\ Shandong Cancer Hospital and Institute, Shandong First Medical University and Shandong Academy of Medical Sciences, \\ Jinan, China
}

Purpose: The purpose of this study was to identify aberrant long non-coding RNAs (IncRNAs) and explore the predictive value of IncRNA expression patterns on the risk of brain metastases (BMs) in patients with limited-stage small-cell lung cancer (SCLC).

Patients and Methods: We executed an array of IncRNA and mRNA chip assays to examine the expression in peripheral blood mononuclear cells obtained from SCLC patients with BMs and compared the expression patterns against those from patients without BMs to identify IncRNAs associated with BMs. Validation was performed against clinical data to further confirm the relationship between IncRNAs and BM. Kaplan-Meier analysis was applied to estimate the cumulative incidence of BMs, and differences between the groups were analyzed using the log-rank test.

Results: The expression of 67 IncRNAs (27 upregulated and 40 downregulated) and 47 mRNAs (20 upregulated and 27 downregulated) was significantly different between the BM and non-BM groups (fold change $\geq 2.0, p$-value $\leq 0.05$ ), based on the IncRNA and mRNA chip assays. Four IncRNAs were verified by quantitative real-time polymerase chain reaction (qRT-PCR) to confirm the accuracy of the microarray data, and the results of 11 patient pairs (11 patients with $\mathrm{BM}$ and 11 patients without $\mathrm{BM}$ ) revealed that low LncRNA XR_429159.1 expression was a high-risk factor for BM. Further clinical data showed that the incidence of BM among 25 patients with low-level LncRNA XR_429159.1 expression was 31\% at 1 year, compared with $14.3 \%$ among the 18 patients with high-level LncRNA XR_429159.1 expression ( $p=0.035$ ).

Conclusion: Our present study identified the low-level expression of IncRNA XR_429159.1 as a high-risk factor among BM in patients with limited-stage SCLC. LncRNA XR_429159.1 is a critical molecule that regulates SCLC metastasis, involved in the neuroepithelial transforming gene 1 (NET1) pathway, and serum levels of this IncRNA are significantly associated with the risk of BM.

Keywords: IncRNA, brain metastasis, small cell lung cancer, risk factors, NET 


\section{INTRODUCTION}

The brain is the most common distant metastasis site for patients with small-cell lung cancer (SCLC), and brain metastasis (BM) is an unfavorable prognosis factor, regardless of the disease stage at presentation. At the time of SCLC diagnosis, $\sim 10-25 \%$ of patients suffer from BM, and the probability of BM increases to $50 \%$ among patients who survive as long as 2 years after diagnosis (1-3).

The positive role played by prophylactic cranial irradiation (PCI) in patients with SCLC was established by a meta-analysis in 1999, and PCI has become part of the standard treatment modality used in patients with limited-stage SCLC who respond well to initial treatment. However, the role played by PCI has recently been challenged in patients with stage I limited- and extensive-stage SCLC, with fewer survival benefits observed following PCI based on follow-up magnetic resonance imaging study (4-7). Our retrospective study revealed that patients with completely resected p-stage II-III SCLC and lymphovascular invasion were at the highest risk for $\mathrm{BM}$, whereas the incidence of BM among patients with p-stage I SCLC was <10\% (8). Therefore, identifying the subgroup with high-risk factors for BM who may obtain a survival benefit from PCI represents an urgent task.

Numerous RNAs are transcribed from the human genome, and protein-coding sequences represent only a small portion of the total transcripts. Non-coding RNAs (ncRNAs) lack proteincoding potential, although some can produce small, functional peptides. LncRNAs were long considered to be transcriptional noise; however, recent evidence suggests that these molecules play important roles in diverse cellular processes, including those associated with normal development and disease pathogenesis. LncRNAs can be divided into two groups according to length: small ncRNAs, shorter than $200 \mathrm{nt}$, are referred to as microRNAs, whereas longer ncRNAs, $>200 \mathrm{nt}$, are referred to as long noncoding RNAs (lncRNAs). MicroRNAs are a well-characterized group of ncRNAs, able to cause gene silencing effects by regulating messenger RNA (mRNA) degradation and repressing translation repression through RNA interference pathways. In addition, gene expression is more complex when regulated by lncRNAs, often involving multiple mechanisms (9-11). LncRNAs play an oncogenic or a tumor suppressive role. For example, PVT1 is highly correlated in primary human tumors (28). MALAT1 can promote tumorigenesis through the PI3K/AKT pathway (29). PCAT-1 has been implicated in prostate cancer patients (30). In addition, apoptosis induced by anticancer drugs can be reduced PCGEM1 (31). HOTAIR is strongly associated with breast cancer metastasis and patient survival (32). In SCLC, lncRNAs have been correlated with drug resistance, disease progression, and prognosis (12). However, several factors remain unknown regarding the correlation between lncRNA status and BM occurrence in SCLC.

In this study, we used an array of lncRNA and mRNA chip assays to examine the expression levels in peripheral blood mononuclear cells (PBMCs) obtained from SCLC patients to determine the relationships between IncRNAs and BM incidence in patients with SCLC.

\section{MATERIALS AND METHODS}

\section{Patients}

Total RNA was obtained from six SCLC patients with BM and six patients without BM (four men and two women in each group). Subsequently, 11 pairs of patients were enrolled for the validation arm of the current study between June 2015 and October 2015 at Shandong Cancer Hospital and Institute. All patients were treatment-naive at the time of PBMCs collection. This study was approved by the ethical review board. All patients signed an informed consent approved by the Institutional Review Board.

\section{RNA Extraction}

Peripheral blood was acquired in ethylenediaminetetraacetic acid-containing tubes from all SCLC patients. Human PBMCs were isolated with Ficoll-Hypaque gradients. Total RNA was extracted from PBMCs using TRIzol $^{\circledR}$ reagent (Invitrogen, Grand Island, NY, USA).

\section{Microarray Imaging and Data Analysis}

Total RNAs were labeled for the lncRNA+mRNA Human Gene Expression Microarray V4.0 (4×180 K), and each array contained a probe set comprising 40,916 human lncRNA transcripts and 34,235 human mRNAs. The datasets presented in this study have been deposited in the following online repositories under the indicated Accession Number(s) (GEO and GSE161968).

We used a LightCycler 480 PCR System (Roche) to perform quantitative real-time polymerase chain reaction (qRT-PCR), using SYBR Premix Ex Taq ${ }^{\mathrm{TM}}$ (TAKARA). Expression data were normalized against the expression of glyceraldehyde 3-phosphate dehydrogenase (GAPDH), and the relative expression levels of each gene were analyzed using the $2^{-\Delta \Delta \mathrm{Ct}}$ method.

\section{Clinical Validation}

The peripheral blood, treated with ethylenediaminetetraacetic acid to prevent coagulation, was obtained from 43 patients with limited-stage SCLC and initial radical therapy, without PCI treatment, between January 2016 and November 2016. The expression levels of lncRNA XR_429159.1 were determined, and the patients were divided into the high and low expression groups. BM was the primary endpoint, and all necessary information was acquired by follow-up. The incidence of BM was calculated in each group and compared.

\section{Statistical Analysis}

Statistical analysis was performed using SPSS 16.0 (SPSS Inc.). All data are expressed as mean $\pm \mathrm{SD}$. A $p$-value $<0.05$ was considered significant. According to the LNCipedia database, all lncRNAs identified in this study have previously been recognized. Kaplan-Meier analysis was used to estimate the actuarial risk of developing BM. A log-rank test was used to compare between the groups. A two-sided $p$-value $<0.05$ was considered significant. 
A
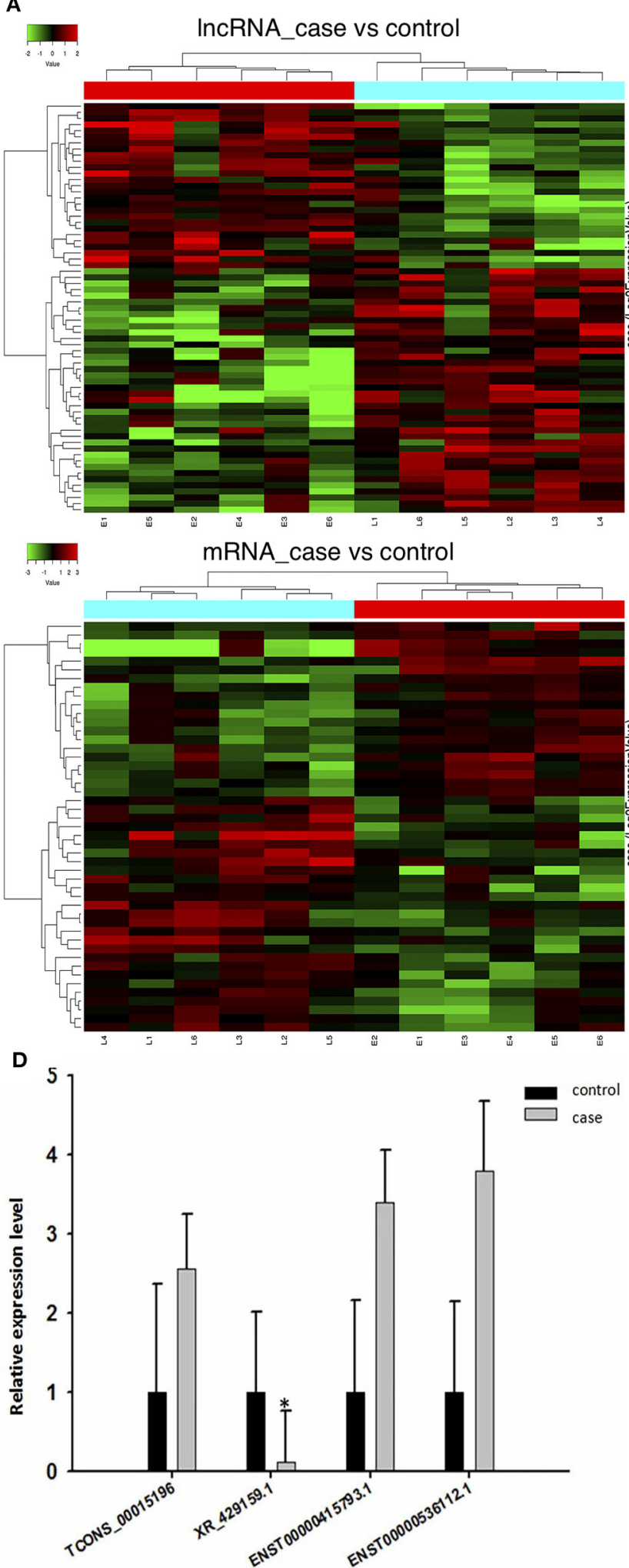

IncRNA expression pattern

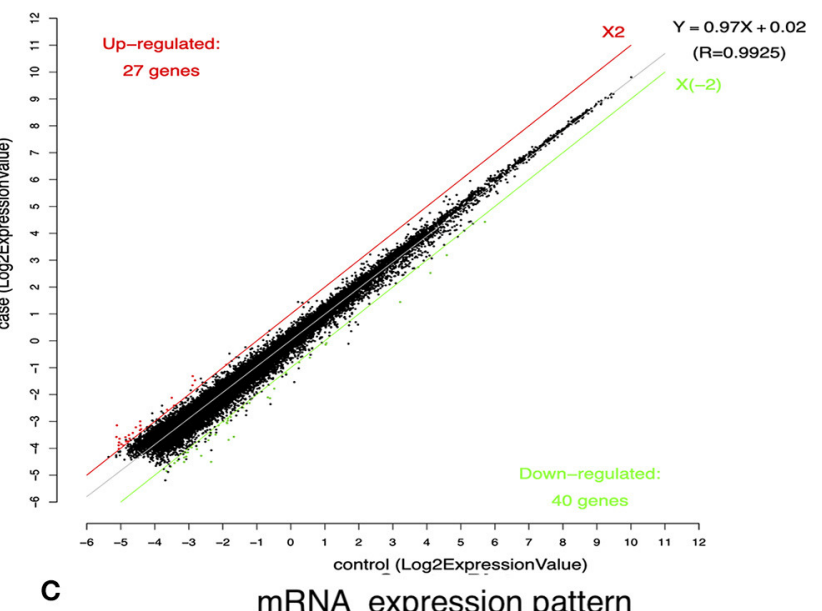

mRNA expression pattern

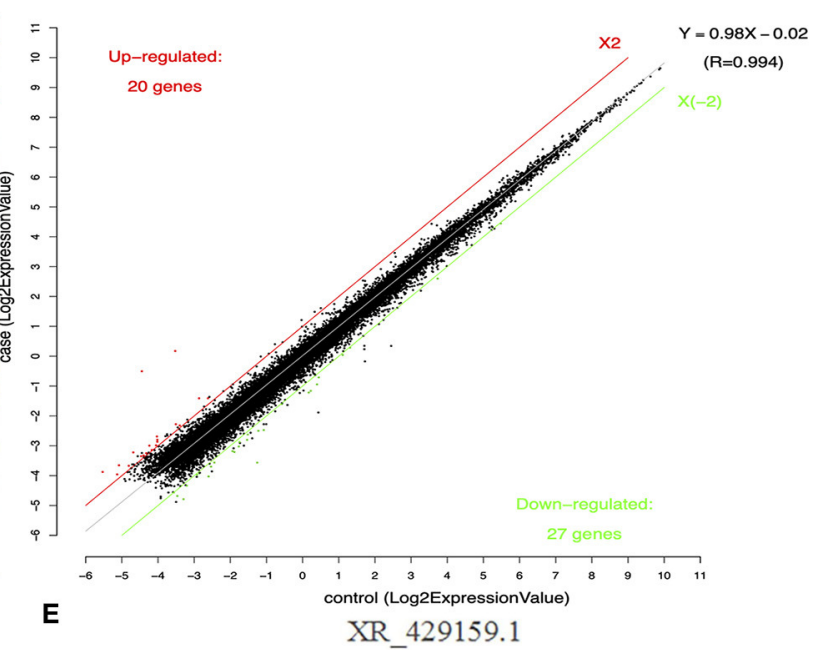

non-BM $\square \mathrm{BM}$

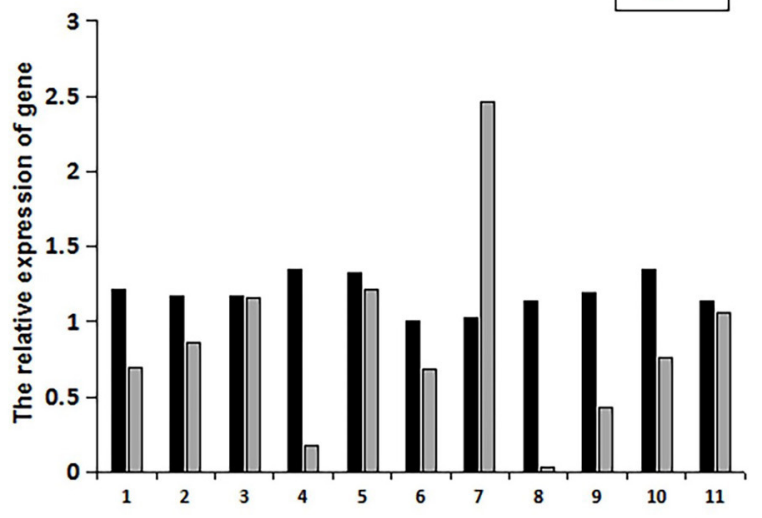

FIGURE 1 | A comprehensive overview of aberrantly expressed InCRNAs and mRNAs in the comparison between BM-SCLC and non-BM-SCLC samples. (A) A total of 67 IncRNAs (27 upregulated and 40 downregulated) and 47 mRNAs (20 upregulated and 27 downregulated) were differentially expressed between BM-SCLC ( $n=$ 6) and SCLC patients without BM $(n=6)$. The scatter plot was used to assess InCRNA (B) and mRNA (C) expression patterns between BM-SCLC patients and controls. X-axis: log2 expression value. Y-axis: log2 expression value. Red: upregulated. Green: downregulated. (D) LncRNA expression levels, as determined by microarray and real-time PCR analyses. (E) The comparison between LncRNA XR_429159.1 levels between 11 pairs of patients with and without BM. ${ }^{*} \mathrm{p}<0.05$. 


\section{RESULT}

\section{Identifying the Candidate IncRNAs Associated With BM}

To confirm the expression levels of lncRNAs and mRNAs in BM-SCLC patients, we performed lncRNA and mRNA microarray analyses on PBMCs obtained from BM-SCLC patients and age- and gender-matched controls (SCLC patients without BM). Cluster 3.0 was used to cluster the lncRNA and mRNA expression data (Figure 1A). Analysis by $t$-test revealed significant differences of up to 2 -fold in the expression levels of certain lncRNAs and mRNAs between the groups $(p<0.05$, Figures 1B,C). As we can see in Figures 1B,C, a total of 67 lncRNAs (27 upregulated and 40 downregulated) and 47 mRNAs (20 upregulated and 27 downregulated) were identified as being differentially expressed in BM-SCLC patients compared with the control patients. The results for the 67 lncRNAs are detailed in Table 1.

\section{IncRNA Expression in BM-SCLC Patients}

To verify differences in lncRNA expression between patients with and without BM-SCLC, qRT-PCR was used to verify the up- and

TABLE 1 | Sixty-seven IncRNAs expressed in BM-SCLC.

\begin{tabular}{|c|c|c|c|c|c|}
\hline IncRNA ID (up) & Probe & $p$ & IncRNA ID (down) & Probe & $p$ \\
\hline ENST00000443576.2 & p4009 & 0.024 & TCONS_00022863 & p19374 & 0.029 \\
\hline ENST00000433357.2 & p16736 & 0.002 & XR_109582.2 & p25097 & 0.017 \\
\hline TCONS_00022087 & p19097 & 0.038 & ENST00000530576.1 & p2682 & 0.037 \\
\hline ENST00000502776.1 & p13887 & 0.030 & TCONS_00008533 & p22324 & 0.017 \\
\hline NR_026881.1 & p25115 & 0.035 & XR_429159.1 & p42511_v4 & 0.013 \\
\hline ENST00000442829.1 & p9753 & 0.012 & ENST00000509873.1 & p12911 & 0.031 \\
\hline ENST00000547326.1 & p3297 & 0.019 & TCONS_00014251 & p23618 & 0.020 \\
\hline TCONS_00015196 & p24068 & 0.004 & RNA33685|snoRNA_scaRNA_281_75 & RNA33685|snoRNA_scaRNA_281_75 & 0.016 \\
\hline TCONS_00012509 & p23458 & 0.016 & ENST00000547777.1 & p3643 & 0.013 \\
\hline ENST00000569037.1 & p6036 & 0.045 & ENST00000599524.1 & p8239 & 0.012 \\
\hline XR_428476.1 & p41744_v4 & 0.001 & ENST00000552511.1 & p34336_v4 & 0.021 \\
\hline TCONS_00010550 & p22837 & 0.007 & XR_426705.1 & p39308_v4 & 0.045 \\
\hline TCONS_00019442 & p18518 & 0.011 & ENST00000536112.1 & p34230_v4 & 0.044 \\
\hline ENST00000441205.1 & p9450 & 0.003 & ENST00000596996.1 & p8237 & 0.043 \\
\hline TCONS_00026648 & p20573 & 0.035 & ENST00000596705.1 & p34609_v4 & 0.004 \\
\hline ENST00000432973.1 & p6336 & 0.020 & ENST00000560026.1 & p5053 & 0.003 \\
\hline ENST00000452503.1 & p186 & 0.018 & TCONS_00022023 & p19047 & 0.007 \\
\hline XR_244656.1 & p40400_v4 & 0.000 & ENST00000509641.2 & p12309 & 0.046 \\
\hline XR_244697.1 & p40446_v4 & 0.011 & ENST00000415793.1 & p8562 & 0.027 \\
\hline TCONS_00024667 & p19899 & 0.027 & XR_426704.1 & p39309_v4 & 0.019 \\
\hline uc001ugl.3 & p25845 & 0.039 & ENST00000607996.1 & p38259_v4 & 0.029 \\
\hline LIT3591 & p28883 & 0.003 & ENST00000434996.1 & p11660 & 0.004 \\
\hline NR_109815.1 & p43698_v4 & 0.003 & XR_429229.1 & p42634_v4 & 0.042 \\
\hline TCONS_00013445 & p23782 & 0.005 & ENST00000599993.1 & p8236 & 0.018 \\
\hline uc010nlv.1 & p26558 & 0.006 & ENST00000416061.1 & p17015 & 0.006 \\
\hline TCONS_00020666 & p18986 & 0.041 & ENST00000435749.1 & p15383 & 0.048 \\
\hline \multirow[t]{14}{*}{ ENST00000454882.2 } & p14463 & 0.008 & ENST00000509350.1 & p16341 & 0.042 \\
\hline & & & ENST00000598981.1 & p29006 & 0.018 \\
\hline & & & ENST00000448195.1 & p15382 & 0.018 \\
\hline & & & ENST00000610044.1 & p37042_v4 & 0.048 \\
\hline & & & TCONS_00021347 & p18641 & 0.041 \\
\hline & & & XR_246000.1 & p42510_v4 & 0.008 \\
\hline & & & TCONS_00014250 & p23617 & 0.008 \\
\hline & & & TCONS_00022866 & p19378 & 0.026 \\
\hline & & & ENST00000593653.1 & p34611_v4 & 0.010 \\
\hline & & & ENST00000607857.1 & p38428_v4 & 0.042 \\
\hline & & & ENST00000420598.1 & p383 & 0.045 \\
\hline & & & NR_046095.1 & p33675 & 0.032 \\
\hline & & & ENST00000459433.1 & p38665_v4 & 0.033 \\
\hline & & & ENST00000504520.1 & p12349 & 0.009 \\
\hline
\end{tabular}


downregulation of IncRNA expression between the groups. Based on the potential pathways predicted by the Kyoto Encyclopedia of Genes and Genomes (KEGG) analysis, eight lncRNAs were identified for further validation. Then, four lncRNAs with several differences were selected to confirm the reliability of the microarray data (the forward and reverse primers used for each gene are shown in Table 2). IncRNA XR_429159.1 was validated, demonstrating an 8.33-fold difference in the expression levels between the groups, which was a more considerable difference than the remaining three lncRNAs (Figure 1D).

To verify the relationship between reduced lncRNA XR_429159.1 expression and an increased risk of BM, we collected 11 pairs of patients, matched for clinical data, gender, and age. The results obtained between these patient pairs were consistent with our previous analysis. As shown in Figure 1E,

TABLE 2 | The primers of four IncRNAs.

\begin{tabular}{ll}
\hline IncRNA & Real-time PCR primers \\
\hline Inc196-F2 & GGACACAGCAGATAGCAGACC \\
Inc196-R2 & CCACAAGGAAGCCATTGAGACA \\
Inc159-F1 & GCTGGAGGCTGACAACAAC \\
Inc159-R1 & ACCTGAAGTGAAAAGGAAGAGAAGA \\
Inc793-F1 & AGGAGTGGGCTITGTGGAT \\
Inc793-R1 & CTCTAAGTGAAGGAATGTTGTCGTC \\
Inc112-F2 & GGCTITAGACCAGGAGACTGTG \\
Inc112-R2 & TCCAGCGAGTGATAGTGGGT
\end{tabular}

the expression of lncRNA XR_429159.1 was more significantly reduced in patients with $\mathrm{BM}$ than in patients without $\mathrm{BM}$, for most pairs, particularly the fourth pair $(\sim 8$-fold difference in the expression) and the eighth pair ( $\sim 30$-fold difference in the expression). In the seventh pair, the expression level of lncRNA XR_429159.1 was higher in the BM patient than in the control patient; we believe that this may be due to specimen degradation during transport. As shown in Figure 2, to verify this result, we recruited 20 pairs of patients, and the expression of lncRNA XR_429159.1 in 15 patients was more significantly reduced in patients with BM than in patients without BM.

\section{The Role of IncRNA XR_429159.1 Expression Levels for Predicting BM}

The clinical characteristics of 43 patients are detailed in Table 3. The last follow-up was conducted in August 2018. Among these 43 patients with limited-stage SCLC, 15 patients (15/43, 34.9\%) developed BM. The level of lncRNA XR_429159.1 expression increased in 18 (41.9\%) patients and decreased in $25(58.1 \%)$ patients. Three (16.7\%) patients developed BM in the high-level group, and $12(42.9 \%)$ patients developed BM in the low-level group. The development time of BM ranged from 7.0 to 26.3 months, with a median time to BM development of 18.0 months, which was determined from the date of diagnosis. The actuarial risk of developing BM at 1 year was $14.3 \%$ in patients with highlevel lncRNA XR_429159.1 compared with 31.0\% in the low-level group, which represented a significant difference, with a $p$-value of 0.035 (Figure 3).

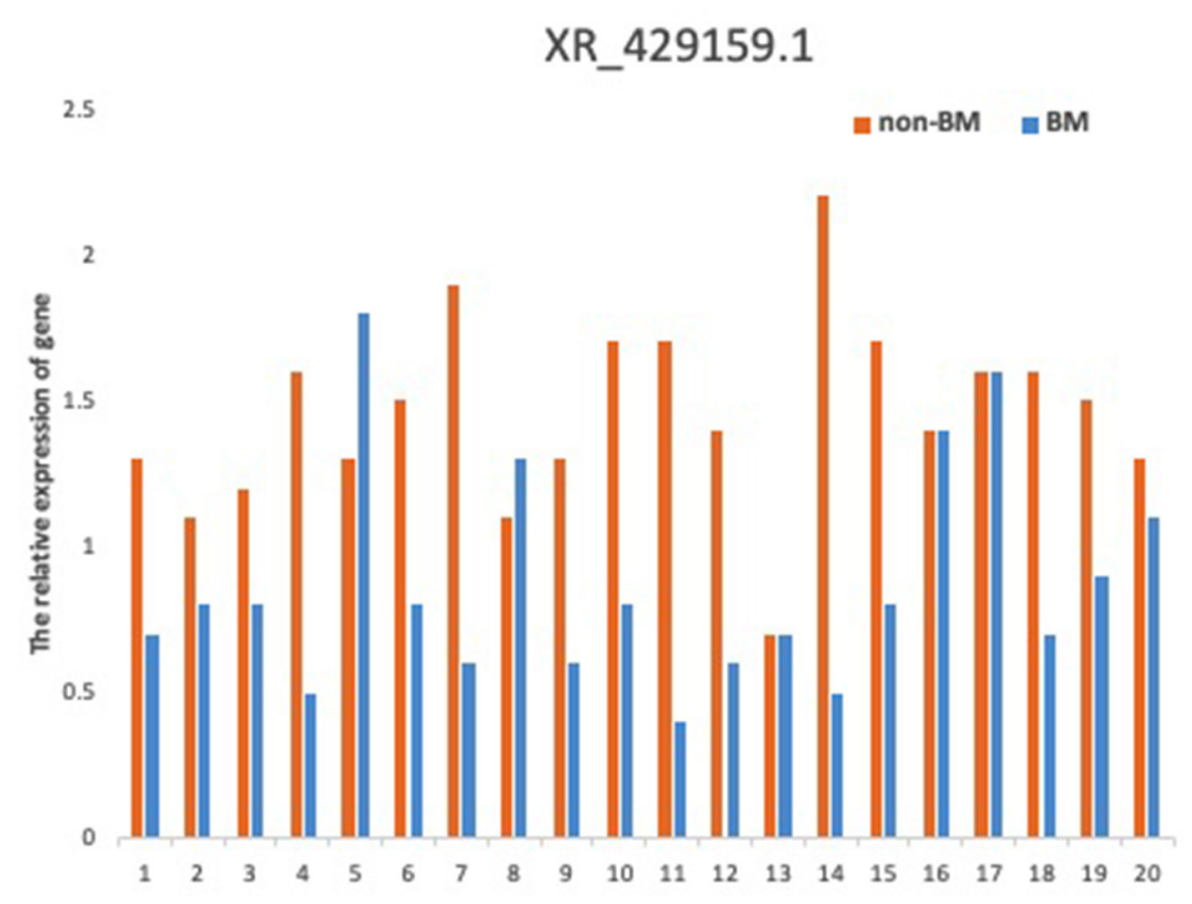

FIGURE 2 | The comparison between LncRNA XR_429159.1 levels between 20 pairs of patients with and without BM. 
TABLE 3 | The characteristics of 43 patients for clinical validation.

\begin{tabular}{lcccc}
\hline & Total & Up (n, \%) & Down $(\boldsymbol{n}, \%)$ & $\boldsymbol{p}$ \\
\hline Age (years) & & & & \\
$<60$ & 11 & $5(27.8)$ & $6(24.0)$ & 0.779 \\
$\geq 60$ & 32 & $13(72.2)$ & $19(76.0)$ & \\
Gender & & & & \\
Male & 34 & $14(77.8)$ & $20(80.0)$ & 0.86 \\
Female & 9 & $4(22.2)$ & $5(20.0)$ & \\
Smoking status & & & & \\
Yes & 29 & $13(72.2)$ & $12(48.0)$ & 0.57 \\
No & 14 & $5(27.8)$ & $13(52.0)$ & \\
Stage & & & & \\
I & 12 & $6(33.3)$ & $6(24.0)$ & 0.763 \\
II & 25 & $10(55.6)$ & $15(60.0)$ & \\
III & 6 & $2(11.1)$ & $4(16.0)$ & \\
Treatment & & & & \\
S+C/R & 17 & $5(27.8)$ & $12(48.0)$ & 0.181 \\
C+R & 26 & $13(72.2)$ & $13(52.0)$ & \\
\end{tabular}

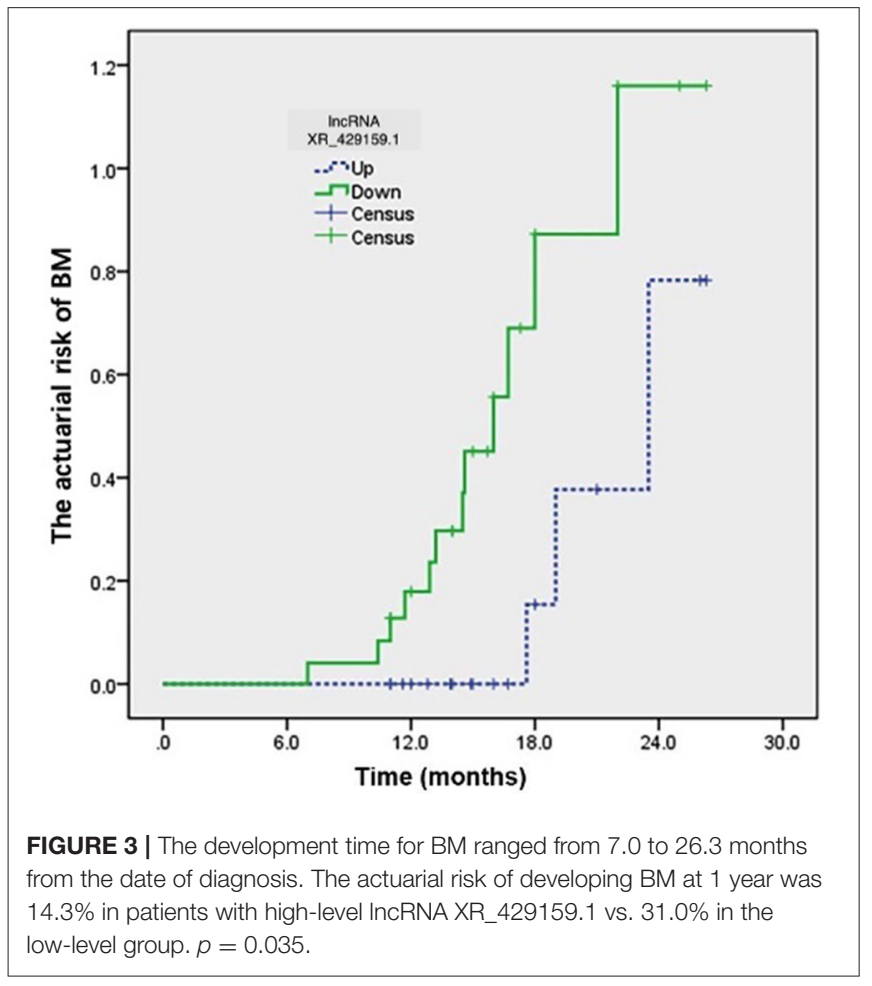

\section{Predictive Signaling Pathway}

The relationship between the expression levels of lncRNAs and $\mathrm{BM}$ was further explored by identifying the potential signaling pathways associated with lncRNA XR_429159.1 based on analysis using the KEGG signaling pathway database. As shown in Figure 4, KEGG analysis showed that lncRNA might be related to ATP-binding cassette $(\mathrm{ABC})$ transporters, cell adhesion molecules, glucose metabolisms, and signal transduction pathways. We identified all genes $10 \mathrm{kbp}$ upstream and downstream of differentially expressed lncRNAs to identify the potential co-expression target genes and action sites. As shown in Table 4, 15 differentially expressed lncRNAs and co-expressed mRNAs were identified. Comparisons between expression levels in patients with $\mathrm{BM}$ and those without $\mathrm{BM}$ revealed that the differentially expressed lncRNA XR_429159.1 might be associated with the neuroepithelial transforming gene 1 (NET1) pathway, which may represent a potential target pathway for preventing SCLC BM.

\section{DISCUSSION}

In the present study, the genome-wide expression patterns of lncRNAs and mRNAs were examined in PBMCs obtained from six BM-SCLC patients and six controls, and these expression patterns were validated using clinical data. LncRNA XR_429159.1 was significantly downregulated in extensive-stage SCLC patients with BM compared with patients with limitedstage SCLC. The correlation between lncRNA XR_429159.1 expression and $\mathrm{BM}$ was further explored in 11 patient pairs, in which limited-stage SCLC patients were matched with extensivestage SCLC patients with BM. The results showed that a decrease in the expression level of lncRNA XR_429159.1 was associated with the occurrence of BM. To further verify these findings, 43 patients with limited-stage SCLC were closely followed, and $58.1 \%$ of the patients were found to have low lncRNA XR_429159.1 expression. The 1-year BM rate for the low expression LncRNA XR_429159.1 group was significantly higher than that for the high expression group $(p=0.035)$. Four patients ( $\geq 70$ years and stage I) did not receive PCI. Two patients in the low lncRNA XR_429159.1 expression group of these four patients developed BM. We confirmed that low-level lncRNA XR_429159.1 was the most significant risk factor for BM among patients with limited-stage SCLC. Further exploration and analysis suggested that lncRNA may be related to the NET1 pathway. This study may help to identify patients at high risk of BM who may acquire a survival benefit from PCI treatment. Additional studies are planned to reveal the molecular mechanisms and biological functions through which lncRNA XR_429159.1 functions in the pathogenesis of BM-SCLC.

LncRNAs can be found in both the nucleus and cytoplasm, and most $\operatorname{lncRNAs}$ located in the nucleus have been found to play important roles in gene expression (13). The abnormal expression of lncRNAs has been associated with the pathogenesis of many diseases, particularly carcinogenesis and the development of malignant tumors $(10,14)$. Han et al. showed that lncRNA H19 could activate the Janus kinase (JAK)/signal transducer and activator of transcription (STAT) pathway to promote the expression of Stat3 and c-Myc, which play vital roles in the progression of glioblastoma (15). The IncRNA MALAT1 acts through the miR-145-5p/A-kinase anchoring protein 12 (AKAP12) axis to influence prostate cancer therapy (16). Small nucleolar RNA host gene 15 (SNHG15) has been shown to provide a potential therapeutic effect through the regulation of CDK14 protein by sponging miR-486 in non-small-cell lung cancer (NSCLC) patients (17). 


\section{Significant Enriched KEGG_PATHWAY.pathway Terms (Top 20)}

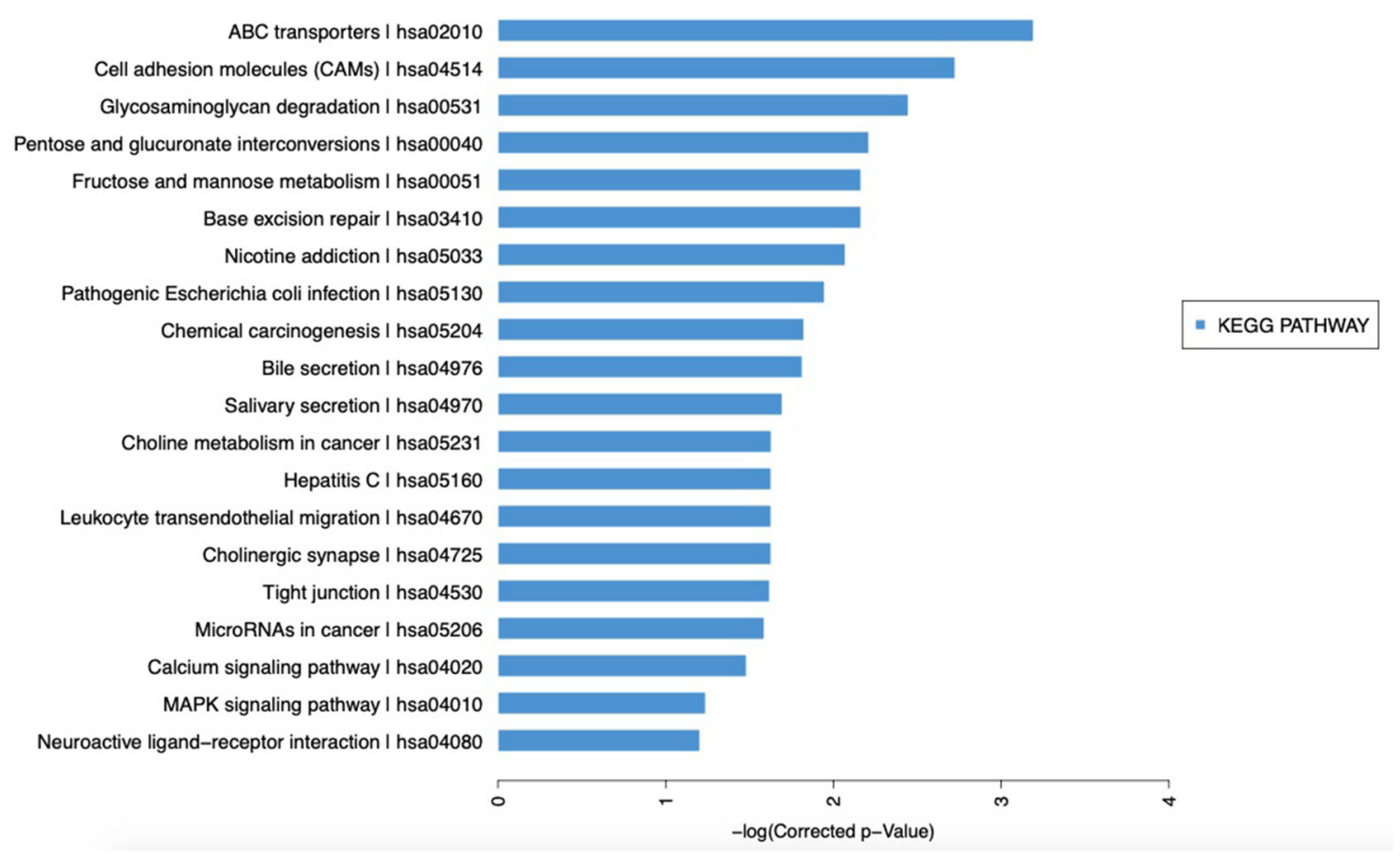

FIGURE 4 | Significantly enriched KEGG pathways. KEGG analysis showed that IncRNA might be related to ABC transporters, cell adhesion molecules, glucose metabolism, and signal transduction pathways.

TABLE 4 | Predictive signaling pathway.

\begin{tabular}{lllr}
\hline IncRNA & mRNA & Target gene & Correlation \\
\hline ENSG00000234773.2 & A_33_P3210085 & NET1 & 0.901010299 \\
ENSG00000232274.1 & A_23_P21785 & NSUN3 & 0.921705135 \\
XR_246000.1 & A_33_P3369716 & InC-APBA2-1 & 0.904952848 \\
XR_429159.1 & A_21_P0011399 & NET1 & 0.926553947 \\
ENSG00000251301.2 & A_33_P3369716 & LOC100507195 & 0.939780957 \\
XR_246000.1 & A_33_P3369716 & LOC100507195 & $5.20 \mathrm{E}-05$ \\
ENSG00000232274.1 & A_33_P3361067 & ABCG2 & 0.991972956 \\
XR_426705.1 & A_21_P0010623 & LINC01359 & 0.951285719 \\
XR_426704.1 & A_21_P0010623 & LINC01359 & 0.989767777 \\
XR_244697.1 & A_19_P00320972 & STMN1 & 0.97878916 \\
XR_426705.1 & A_21_P0010621 & LINC01359 & -0.94305485 \\
XR_426704.1 & A_21_P0010621 & LINC01359 & $2.59 E-10$ \\
ENSG00000257497.1 & A_23_P111662 & ABCB5 & $1.99 E-06$ \\
XLOC_007255 & A_33_P3339625 & IL17C & $8.68 E-10$ \\
ENSG00000233064.2 & A_32_P19539 & TMEM56 & $3.26 E-08$ \\
\hline
\end{tabular}

Recently, some studies have shown that the abnormal expression of lncRNAs in SCLC may reflect disease progression and predict clinical outcomes. For example, a study indicated that HOXA distal transcript antisense RNA (HOTTIP), which is highly expressed in SCLC tissues, might affect cell proliferation and cell cycle regulation in SCLC patients, promoting SCLC tumorigenesis (18). Another lncRNA, taurine upregulated gene
1 (TUG1), is involved in cell apoptosis, cell cycle regulation, cell proliferation, migration, invasion, and chemoresistance (19). Moreover, TUG1 was upregulated in SCLC tissues and was associated with both the clinical staging and overall survival (OS) of patients with SCLC. Similarly, PVT1, which was overexpressed in SCLC tissues, has also been associated with malignant status and poor prognosis in SCLC patients (20). However, the 
expression patterns, potential targets, and functions of lncRNAs and their effects on disease development and pathogenesis remain unclear.

LncRNA may affect SCLC through multiple pathways; however, the underlying mechanisms remain unclear. Zhang et al. found that the lncRNA SBF2 antisense RNA 1 (SBF2-AS1) acted as an oncogenic lncRNA in SCLC (21), with increased expression in SCLC cell lines. In addition, high expression of SBF2-AS1 was associated with poor survival, distant metastasis, and lymph node metastasis. Another study indicated that lncRNAs might promote cancer cell stemness in SCLC (22). The overexpression of LncRNA cancer susceptibility 11 (CASC11) increased the proportion of CD133+ cells among SCLC cell lines. Increased cancer stem cells could promote distant metastasis and drug resistance. HOTTIP enhanced chemoresistance in SCLC cells by regulating $\mathrm{B}$-cell lymphoma 2 (BCL-2), increasing the expression of the anti-apoptotic factor BCL-2 and miR-216a (23). In our present study, a low level of lncRNA XR_429159.1 was correlated with $\mathrm{BM}$, further suggesting that the gene regulated by this lncRNA was a suppressor gene.

Because PBMCs are immune cells, we hypothesize that the whole-body immune response can influence BM. Recent studies have shown that immunotherapy is effective for the treatment of SCLC BM. An updated analysis of the CASPIAN trial showed a prolonged, 3.2-month (12.0 vs. 8.8 months) increase in OS in the durvalumab plus platinum-etoposide group compared with that in the platinum-etoposide alone group, with a hazard ratio (HR) of 0.69 (95\% CI, 0.35-1.31) (24).

The CASPIAN subgroup analyses examining BM demonstrated for the first time that durvalumab, a programmed death-ligand 1 (PD-L1) inhibitor, can significantly improve OS in SCLC patients with BM. However, few studies have examined the effects of PBMCs on BM. We hypothesized that changes in the regulatory factors associated with PBMCs would lead to changes in immune status, affecting the occurrence of BM. However, because PBMCs are regulated by several factors, the relationships among these factors remain unclear. LncRNA expression was correlated with BM occurrence in some studies (25-27). Therefore, we aimed to explore whether specific molecular markers could be identified in PBMCs that might affect the development of BM, such as lncRNA XR_429159.1.

However, this study has some limitations. Firstly, due to the early development of this study, the included sample size was relatively small, and the subsequent survival data obtained for the clinical sample validation remain immature, which made the impact of lncRNAs on survival challenging to analyze further. Secondly, only preliminary detection and clinical verification have been performed in this study, and no experiments have

\section{REFERENCES}

1. Govindan R, Page N, Morgensztern D, Read W, Tierney R, Vlahiotis A, et al. Changing epidemiology of small-cell lung cancer in the United States over the last 30 years: analysis of the surveillance, epidemiologic, and end results database. J Clin Oncol. (2006) 24:4539-44. doi: 10.1200/JCO.2005.04.4859 been performed to further analyze the potential mechanisms through which this lncRNA affects BM, such as its correlation with the NET1 pathway. Thirdly, lncRNAs are diverse and have complex functions. Although some potentially regulated genes were identified based on the second-generation sequencing results, other lncRNAs that may have a potential impact on the development of BM in SCLC cannot be excluded, and only the potential mechanisms of action can be explored based on these predicted results. Therefore, the selection of multiple lncRNAs may help clarify the mechanism of SCLC BM and may provide a reference for clinical drug development.

\section{CONCLUSION}

In summary, this study described the expression of lncRNAs in patients with BM-SCLC using an RNA microarray method. These findings suggested that SCLC patients with low expression levels of lncRNA XR_429159.1 were at high risk for BM. LncRNA XR_429159.1 is an important molecule that may regulate SCLC metastasis, potentially through the NET pathway, and serum levels of this lncRNA were significantly associated with the risk of BM.

\section{DATA AVAILABILITY STATEMENT}

My data is publicly available, the number and link are GSE161968 and https://www.ncbi.nlm.nih.gov/geo/query/acc. cgi? acc $=$ GSE161968.

\section{ETHICS STATEMENT}

This study was approved by The Ethical Review Board of Shandong Cancer Hospital and Institute and complied with the Helsinki declaration. Written informed consent was obtained from all participants.

\section{AUTHOR CONTRIBUTIONS}

JL and WJ drafted the manuscript. XZ and WJ edited and finalized the manuscript. HZ prepared the figures. All authors contributed to the article and approved the submitted version.

\section{FUNDING}

This study was supported by the National Natural Science Foundation of China under Grant Number 81972862 (to HZ).

2. Socha J, Kepka L. Prophylactic cranial irradiation for small-cell lung cancer: how, when and for whom? Expert Rev Anticancer Ther. (2012) 12:50517. doi: 10.1586/era.12.19

3. Seute T, Leffers P, Ten Velde GP, Twijnstra A. Neurologic disorders in 432 consecutive patients with small cell lung carcinoma. Cancer. (2004) 100:8016. doi: $10.1002 /$ cncr.20043 
4. Auperin A, Arriagada R, Pignon JP, Pechoux CL, Gregor A, Stephens RJ, et al. Prophylactic cranial irradiation for patients with small-cell lung cancer in complete remission. Prophylactic cranial irradiation overview collaborative group. N Engl J Med. (1999) 341:476-84. doi: 10.1056/NEJM199908123410703

5. Zhu H, Guo H, Shi F, Zhu K, Luo J, Liu X, et al. Prophylactic cranial irradiation improved the overall survival of patients with surgically resected small cell lung cancer, but not for stage I disease. Lung Cancer. (2014) 86:334-8. doi: 10.1016/j.lungcan.2014.09.019

6. Xu J, Yang H, Fu X, Jin B, Lou Y, Zhang Y, et al. Prophylactic cranial irradiation for patients with surgically resected small cell lung cancer. J Thorac Oncol. (2017) 12:347-53. doi: 10.1016/j.jtho.2016.09.133

7. Wu AJ, Gillis A, Foster A, Woo K, Zhang ZG, Gelblum DY, et al. Patterns of failure in limited-stage small cell lung cancer: implications of TNM stage for prophylactic cranial irradiation. Radiother Oncol. (2017) 125:1305. doi: 10.1016/j.radonc.2017.07.019

8. Zhu H, Bi Y, Han A, Luo J, Li M, Shi F, et al. Risk factors for brain metastases in completely resected small cell lung cancer: a retrospective study to identify patients most likely to benefit from prophylactic cranial irradiation. Radiat Oncol. (2014) 9:216. doi: 10.1186/1748-717X-9-216

9. Gibb EA, Brown CJ, Lam WL. The functional role of long non-coding RNA in human carcinomas. Mol Cancer. (2011) 10:38. doi: 10.1186/1476-4598-10-38

10. Peng WX, Koirala P, Mo YY. LncRNA-mediated regulation of cell signaling in cancer. Oncogene. (2017) 36:5661-67. doi: 10.1038/onc.2017.184

11. Zhang F, Gao C, Ma XF, Peng XL, Zhang RX, Kong DX, et al. Expression profile of long noncoding RNAs in peripheral blood mononuclear cells from multiple sclerosis patients. CNS Neurosci Ther. (2016) 22:298305. doi: $10.1111 / \mathrm{cns} .12498$

12. Chen W, Hang Y, Xu W, Wu J, Chen L, Chen J, et al. BLACAT1 predicts poor prognosis and serves as oncogenic lncRNA in small-cell lung cancer. J Cell Biochem. (2018) 1-7. doi: 10.1002/jcb.27548

13. Derrien T, Johnson R, Bussotti G, Tanzer A, Djebali S, Tilger H, et al. The GENCODE $\mathrm{v} 7$ catalog of human long noncoding RNAs: analysis of their gene structure, evolution, and expression. Genome Res. (2012) 22:177589. doi: $10.1101 /$ gr.132159.111

14. Wu GC, Pan HF, Leng RX, Wang DG, Li XP, Li XM, et al. Emerging role of long noncoding RNAs in autoimmune diseases. Autoimmun Rev. (2015) 14:798-805. doi: 10.1016/j.autrev.2015.05.004

15. Han CL, Ge M, Liu YP, Zhhao XM, Wang KL, Chen N, et al. LncRNA H19 contributes to hippocampal glial cell activation via JAK/STAT signaling in a rat model of temporal lobe epilepsy. J Neuroinflammation. (2018) 15:103. doi: 10.1186/s12974-018-1139-Z

16. Xue D, Lu H, Xu HY, Zhou CX, He XZ. Long noncoding RNA MALAT1 enhances the docetaxel resistance of prostate cancer cells via miR-1455p-mediated regulation of AKAP12. J Cell Mol Med. (2018) 22:322337. doi: $10.1111 / \mathrm{jcmm} .13604$

17. Jin $\mathrm{B}$, Jin $\mathrm{H}, \mathrm{Wu} \mathrm{HB}, \mathrm{Xu} J$ J, Li B. Long non-coding RNA SNHG15 promotes CDK14 expression via miR-486 to accelerate non-small cell lung cancer cells progression and metastasis. J Cell Physiol. (2018) 233:716472. doi: $10.1002 / j c p .26543$

18. Sun Y, Zhou Y, Bai Y, Wang Q, Bao J, Luo Y, et al. A long noncoding RNA HOTTIP expression is associated with disease progression and predicts outcome in small cell lung cancer patients. Mol Cancer. (2017) 16:162. doi: 10.1186/s12943-017-0729-1

19. Niu Y, Ma F, Huang W, Fang S, Li N, Wei T, et al. Long non-coding RNA TUG1 is involved in cell growth and chemoresistance of small cell lung cancer by regulating LIMK2b via EZH2. Mol Cancer. (2017) 16:5. doi: 10.1186/s12943-016-0575-6
20. Huang C, Liu S, Wang H, Zhang Z, Yang Q, Gao F. LncRNA PVT1 overexpression is a poor prognostic biomarker and regulates migration and invasion in small cell lung cancer. Am J Transl Res. (2016) 8:5025-34.

21. Zhang Y, Li Y, Han L, Zhang P, Sun S. SBF2-AS1: an oncogenic lncRNA in small-cell lung cancer. J Cell Biochem. (2019) 120:154228. doi: $10.1002 /$ jcb. 28809

22. Fu $Y$, Zhang $P$, Nan $H$, Lu $Y$, Zhao $J$, Yang $M$, et al. LncRNA CASC11 promotes TGF-beta1, increases cancer cell stemness and predicts postoperative survival in small cell lung cancer. Gene. (2019) 704:916. doi: 10.1016/j.gene.2019.04.019

23. Sun $\mathrm{Y}, \mathrm{Hu} \mathrm{B}$, Wang $\mathrm{Q}$, Ye $\mathrm{M}$, Qiu Q, Zhou $\mathrm{Y}$, et al. Long non-coding RNA HOTTIP promotes BCL-2 expression and induces chemoresistance in small cell lung cancer by sponging miR-216a. Cell Death Dis. (2018) 9:85. doi: 10.1038/s41419-017-0113-5

24. Chen Y, Paz-Ares LG, Dvorkin M, Trukhim D, Reinmuth N, Garassino MC. First-line durvalumab plus platinum-etoposide in extensive-stage (ES)-SCLC (CASPIAN): impact of brain metastases on treatment patterns and outcomes. J Clin Oncol. (2020) 38:9068. doi: 10.1200/JCO.2020.38.15_suppl.9068

25. Yunhe $\mathrm{Lu}$, Lei Chen, Liangdong Li, Yiqun Cao. Exosomes derived from brain metastatic breast cancer cells destroy the blood-brain barrier by carrying IncRNA GS1-600G8.5. Biomed Res Int. (2020) 2020:7461727. doi: 10.1155/2020/7461727

26. Lingxia Liu, $\mathrm{Yu}$ Zhang, Jun $\mathrm{Lu}$. The roles of long noncoding RNAs in breast cancer metastasis. Cell Death Dis. (2020) 11:749. doi: 10.1038/s41419-020-02954-4

27. Shouyu Wang, Ke Liang, Qingsong Hu, et al. JAK2-binding long noncoding RNA promotes breast cancer brain metastasis. J Clin Invest. (2017) 127:4498515. doi: 10.1172/JCI91553

28. Tseng YY, Moriarity BS, Gong W, Akiyama R, Tiwari A, Kawakami H, et al. PVT1 dependence in cancer with MYC copy-number increase. Nature. (2014) 512:82-6. doi: 10.1038/nature13311

29. Liu J, Peng WX, Mo YY, Luo D. MALAT1-mediated tumorigenesis. Front Biosci (Landmark Ed). (2017) 22:66-80. doi: 10.2741/4472

30. Prensner JR, Iyer MK, Balbin OA, Dhanasekaran SM, Cao Q, Brenner JC, et al. Transcriptome sequencing across a prostate cancer cohort identifies PCAT-1, an unannotated lincRNA implicated in disease progression. Nat Biotechnol. (2011) 29:742-9. doi: 10.1038/nbt.1914

31. Fu X, Ravindranath L, Tran N, Petrovics G, Srivastava S. Regulation of apoptosis by a prostate-specific and prostate cancerassociated noncoding gene, PCGEM1. DNA Cell Biol. (2006) 25:135-41. doi: 10.1089/dna.2006.25.135

32. Gupta RA, Shah N, Wang KC, Kim J, Horlings HM, Wong DJ, et al. Long non-coding RNA HOTAIR reprograms chromatin state to promote cancer metastasis. Nature. (2010) 464:1071-6. doi: 10.1038/nature 08975

Conflict of Interest: The authors declare that the research was conducted in the absence of any commercial or financial relationships that could be construed as a potential conflict of interest.

Copyright (๑) $2021 \mathrm{Li}$, Jing, Jia, Zhai, Zhu and Yu. This is an open-access article distributed under the terms of the Creative Commons Attribution License (CC BY). The use, distribution or reproduction in other forums is permitted, provided the original author(s) and the copyright owner(s) are credited and that the original publication in this journal is cited, in accordance with accepted academic practice. No use, distribution or reproduction is permitted which does not comply with these terms. 